\title{
Las epistemologías de la política educativa: vigilancia y posicionamiento epistemológico del investigador en política educativa
}

\section{The epistemology of educational policies: monitoring and epistemological positioning of the educational policies researcher}

César G. Tello*

Resumen: La finalidad de este trabajo consiste en desarrollar la categoría de las epistemologías de la política educativa como un enfoque que propicia la práctica de la vigilancia epistemológica en la investigación en el campo. Esto conlleva necesariamente a realizar por parte del investigador un meta-análisis de su propia investigación Simultáneamente el enfoque de las epistemologías de la política educativa también se convierte en un esquema analítico apto para los estudios epistemológicos en el campo, más allá del uso que cada investigador realice para su propia vigilancia epistemológica en el proceso de desarrollo de su investigación. Este esquema de análisis surge por la preocupación de observar en una parte significativa de investigaciones en nuestra región la ausencia de posicionamientos epistemológicos en la investigación en política educativa, observando que la producción del conocimiento en el campo se despliega desde una combinación metodológica que no se sustenta en perspectivas epistemológicas, y así, la investigación se presenta como neutral e independiente de la realidad social y cultural.

Palabras clave: Política Educativa. Epistemología. Vigilancia Epistemológica.

Resumo: O objetivo deste artigo é desenvolver a categoria epistemologias da política educacional como uma abordagem que incentiva a prática da vigilância epistemológica nas pesquisas desse campo. Essa categoria leva, necessariamente, o pesquisador a realizar uma meta-análise de sua própria investigação. Simultaneamente, o enfoque das epistemologias da política educacional também se torna um esquema analítico adequado para os estudos epistemológicos do campo, além do uso que cada pesquisador pode realizar para a sua própria vigilância epistemológica no processo de desenvolvimento de sua pesquisa. Este esquema de análise surge da preocupação de que, em uma parte significativa de pesquisas sobre políticas educacionais na América Latina, há a ausência de posicionamentos epistemológicos. A produção de conhecimento no campo se desdobra em uma combinação metodológica que não se fundamenta em perspectivas epistemológicas e, assim, a investigação se apresenta como aparentemente neutra e independente da realidade social e cultural.

Palavras-chave: Política educacional. Epistemologia. Vigilância epistemológica.

*Profesor de la Universidad Nacional de San Martín y Universidad Nacional de Tres de Febrero. E-mail: <cesargeronimotello@yahoo.com.ar> 


\begin{abstract}
The aim of this article is to analyze the category referred to as "epistemology of educational politics" as an approach that fosters the epistemological monitoring in the researches performed in this field. This category necessarily leads the researcher to develop a meta-analysis of his own investigation. Simultaneously, the approach of the epistemology of educational policies also becomes an adequate analytical framework for the epistemological studies of the field, beyond the use of each researcher for his own epistemological monitoring during the development of his research. This analytical framework is derived from the concern that, in a significant amount of the research on educational policies performed in Latin America, there is an absence of epistemological positioning. The production of knowledge in the field unfolds in a methodological combination that is not based on epistemological perspectives, thus, the resulting investigations are presented as neutral and detached from the social and cultural reality.
\end{abstract}

Keywords: Educational policies. Epistemology. Epistemological monitoring.

\title{
Presentación
}

Este artículo tiene por objeto describir y desarrollar la categoría de las epistemologías de la política educativa (TELLO, 2009; 2010; 2011a; 2012) en tanto enfoque que permita desplegar en los investigadores del campo un esquema analítico-conceptual, cuya potencia resida en ejercer la vigilancia epistemológica (BOURDIEU et al., 2008) sobre los propios procesos de investigación, contribuyendo a establecer y hacer explícito el posicionamiento epistemológico del investigador en política educativa. Otro uso que promueve el enfoque de las epistemologías de la política educativa (EEPE) se sitúa en el marco de los estudios epistemológicos del campo. En este caso ya no es el propio investigador que despliega la vigilancia epistemológica sobre su proceso investigativo, sino investigadores que analizan la producción del conocimiento en política educativa ${ }^{1}$. En consecuencia, nuestra propuesta consiste en generar un enfoque que permita reflexionar sobre la producción del conocimiento en el campo de la política educativa desde una perspectiva epistemológica en términos metaanalíticos sobre la investigación, tanto en el primero como en el segundo uso.

De este modo podemos afirmar que el eje principal del EEPE es su dimensión analítica en tanto su intención consiste en la reflexividad epistemológica, en términos de vigilancia epistemológica, en cuanto a la noneutralidad de la producción de conocimiento en el campo. Esta cuestión ha sido analizada y reflexionada suficientemente en el marco de las ciencias sociales por diversos autores (HABERMAS, 1981, 1984; SOUSA SANTOS, 2006, 2009; BOURDIEU et al., 2008; entre otros).

\footnotetext{
${ }^{1}$ Veáse por ejemplo la web de la RelePe (Red Latinoamericana de Estudios Epistemológicos en Política Educativa) en $<$ http://www.relepe.org $>$.
} 
Desde esta perspectiva, consideramos que existen dos dificultades epistemológicas en la producción de conocimiento en política educativa:

La primera, la producción del conocimiento del campo en muchos casos se desarrolla sin hacer explícito el posicionamiento del investigador (Cfr. relevamiento realizado por TELLO; MAINARDES, 2012), así la investigación se lleva a cabo como independiente de la realidad social y cultural, o en el sentido de Morín (2001) sin un trasfondo antropo-etico. Aunque el investigador no tenga esa intencionalidad. Sin embargo, al no hacer explícito su posicionamiento, las investigaciones que hemos relevado en el estudio mencionado anteriormente, connotan un desarrollo y producción de conocimiento en política educativa desde una posición y perspectiva pseudo-neutral o positivista (TELLO; MAINARDES, 2012), esto es: llevar a cabo una investigación desde la posibilidad inexistente de la neutralidad y objetividad valorativa. Claro está, que existen esquemas metodológicos que permiten sostener coherencia y consistencia epistemetodológica ${ }^{2}$, pero sería una falacia sostener la neutralidad del investigador en su proceso de investigación, tanto en la obtención de los resultados como en la difusión de conocimientos, imposibilitando la reflexividad epistemológica ${ }^{3}$ sobre su propio proceso de investigación. En fin, la ausencia de posicionamientos explícitos en la investigación en política educativa atenta contra la solidez y coherencia del proceso de investigación.

La segunda dificultad es que en muchas investigaciones en política educativa hemos observado que se siguen metodologías de autores referenciales sin desarrollar el sustento epistemológico. Mixturando técnicas metodológicas, categorías analíticas de autores y perspectivas epistemológicas que poco tiene que ver entre sí, generando inconsistencia epistemológica en el proceso de investigación.

Por esta razón argumentamos que el EEPE contribuye a la vigilancia epistemológica en términos de Bourdieu et al. (2008), para las dos dificultades mencionadas, considerando que:

\footnotetext{
${ }^{2}$ Definimos la epistemetodología según lo hemos planteado en otro trabajo (TELLO, 2011b), en tanto la metodología es el modo en que se enhebran epistemológicamente los componentes metodológicos. Considerando que los componentes metodológicos sin el sustento y las decisiones epistemológicas del investigador carecen de consistencia. En fin, la cuestión metodológica se simplificó a los efectos de la enseñanza, escindiéndola de su sustento: la epistemología. Así, en los talleres de metodología de la carreras de grado y posgrado en Latinoamérica se re afirman técnicas metodológicas que prescinden de perspectivas epistemológicas extendiéndose a posteriori en las investigaciones en ciencias sociales (Cfr. TELLO, 2011b; BARRIGA; HENRÍQUEZ, 2003), particularmente en el campo que analizamos aquí de políticas educativas.

${ }^{3}$ En términos de Bourdieu la reflexividad epistemológica debe ser entendida como lo que el autor denomina el monismo metodológico. Esta última categoría fue mal interpretada en las lecturas y reflexiones, como un intento de Bourdieu por volver al método deductivo. Por el contrario el sociólogo frances planteaba la necesidad de reflexividad epistemológica en términos de vigilancia epistemológica para desarrollar una investigación sólida y coherente. Otros de los aspectos que incluye en su categoría de reflexividad epistemológica se refiere a la dimensión ética y al compromiso social del investigador con la realidad.
} 
Confrontando continuamente a cada científico con una explicitación crítica de sus operaciones científicas y de los supuestos que implican y obligándolo por este medio a hacer de esta explicitación el acompañante obligado de su práctica y de la comunicación de sus descubrimientos, este < sistema de controles cruzados $>$ tiende a constituir y reforzar sin cesar en cada uno la aptitud de vigilancia epistemológica. (BOURDIEU et al., 2008, p. 115).

En este trabajo nos centramos en el EEPE en tanto despliega la posibilidad de meta-análisis del proceso de investigación del campo, lo cual advierte sobre la coherencia y consistencia que se tiene en el desarrollo de las pesquisas con los tres componentes que constituyen las epistemologías de la política educativa: posicionamiento epistemológico, enfoque epistemológico y perspectiva epistemetodológica, como un modo de "sistema de controles cruzados".

Ahora bien, asumimos para constituir los tres componentes en términos del EEPE como un modo de llevar a cabo la práctica de la vigilancia epistemológica, el enfoque de la sociología política de la educación en perspectiva crítica (TORRES, 1999; BALL, 1997; POPKEWITZ, 1994). Y en este sentido adherimos a los postulados de Bourdieu (2008) en tanto critica el "rigorismo tecnológico" que se sostiene en la rigidez de lo definido y de la representación fija de la realidad.

Finalmente argumentamos que la epistemología de la política educativa es un enfoque/esquema que permite el análisis de la producción de conocimiento del campo, no es en sí misma un posicionamiento, sino el modo de analizar los posicionamientos en términos del propio investigador como esquema facilitador para llevar a cabo la práctica de la vigilancia epistemológica y en segundo lugar para llevar a cabo estudios epistemológicos en política educativa.

\section{Las epistemologías de la política educativa y sus componentes analíticos}

Hemos desarrollado esta categoría - en tanto enfoque - como el conjunto de tres componentes: la posición epistemológica, la perspectiva epistemológica y la perspectiva epistemetodológica. Considerando el EEPE como un modo de reflexionar sobre la investigación en el campo. En este sentido es importante tomar la advertencia de Ana Vitar (2006, p. 26) cuando afirma que: "No debemos confundir los procesos políticos y sociales con las herramientas para pensarlos".

Utilizamos la categoría "Epistemologías" en plural dado que existen diversos posicionamientos y perspectivas epistemológicas para desarrollar y llevar a cabo una investigación en política educativa, esto es: existen diversas epistemologías de la política educativa ${ }^{4}$. Por otro lado, es necesario señalar

\footnotetext{
${ }^{4}$ Sin embargo se debe tener en cuenta que en la producción de conocimiento de cada investigador se tendría que poder observar una epistemología coherente y sólida con los tres componentes del EEPE. Con esto nos referimos a que la perspectiva puede ser, por ejemplo la del pluralismo epistemológico, en la que confluyen varias miradas, pero debe ser clara su posición. Cfr. el ejemplo de S. Ball en Tello y Mainardes (2012).
} 
que empleamos la categoría de política educativa en singular para referirnos al campo teórico, dado que no es nuestra intención tomar la compleja distinción anglosajona entre politics y policy. Considerando que la misma es ambigua en nuestra región al intentar traducirla al español o al portugués Como afirma Cox (2006, p. 3) "no se puede recuperar el sentido del segundo término si no es especificando políticas sectoriales". Por esta razón nos referimos con el término Política Educativa al campo teórico y con Políticas Educativas a la acción política como fenómeno educativo posible de ser investigado.

Habiendo hecho estas aclaraciones conceptuales, definiremos brevemente cada una de los componentes que conforman el EEPE.

Entendemos por Perspectiva Epistemológica para el caso de las investigaciones en política educativa como en lo que en metodología de investigación se denomina la Teoría General, en el sentido de Glaser y Strauss (1967), es decir la cosmovisión que el investigador asume para guiar la investigación. Ejemplo de perspectivas podrían ser el marxismo, neo-marxismo, estructuralismo, pos-estructuralismo, existencialismo, humanismo, positivismo y pluralismo. El Posicionamiento Epistemológico se desprende desde la propia Perspectiva Epistemológica o debería desprenderse en una investigación coherente y consistente. El Posicionamiento epistemológico, esto es la Teoría Sustantiva (GLASER; STRAUSS, 1967) vinculada particularmente al campo de estudio, es decir a las corrientes teóricas "propias" 5 . Este es el eje y la nervadura de la categoría epistemologías de la política educativa, dado que es aquí donde se pone en juego la presencia de la cosmovisión del investigador. Entendiendo la cosmovisión como "un conjunto de presuposiciones (o premisas) que sostenemos acerca de la constitución básica de nuestro mundo." (SIRE, 2004, p. 17). Esto es, no solo modos de leer la realidad, sino los modos de construirla, en términos de reflexividad epistemológica. En este sentido el posicionamiento epistemológico se convierte en el posicionamiento político del investigador. Desde nuestra posición es aquí donde se observa la vinculación con la sociología política de la educación en perspectiva crítica. Podemos mencionar los posicionamientos neoinstitucionalistas, institucionalista, clásico o jurídicolegal, constructivismo político, de la complejidad, eclecticismo, posmoderno, posmodernista, hiperglobalista, escéptico, neoliberal, enfoque transformador, funcionalista, crítico, crítico radical, crítico-analítico, teóricos de la resistencia, crítico-reproductivista, humanista, economicista, etc. El posicionamiento epistemológico reposa sobre la selección que realiza el investigador en cuanto la perspectiva epistemológica con la que desarrollará su investigación. Estos posicionamientos epistemológicos son la adjetivación de las perspectivas

\footnotetext{
${ }^{5}$ Entrecomillamos el término porque como veremos el campo de la política educativa se constituye con la presencia de otros campos y saberes.
} 
epistemológicas. Así un investigador desarrolla una epistemología de la política educativa cuando se sitúa y explicita en su investigación que su análisis parte, por ejemplo, de una perspectiva epistemológica neo-marxista y de un posicionamiento epistemológico vinculado a las teorías de la resistencia ${ }^{6}$.

Una cuestión que debemos destacar es que no hemos incluido en el Posicionamiento Epistemológico ni en las Perspectivas Epistemológicas aquello que denominamos "estudios sobre...", esto es: estudios sobre género y políticas educativas, estudios sobre el discurso, estudio de las instituciones, estudios comparados en educación, etc. Claramente estas no son perspectivas epistemológicas ni traen per se un posicionamiento epistemológico. Existen estudios sobre género y políticas educativas que se realizan desde una perspectiva pluralista, neomarxista o positivista. En este sentido no debemos confundir subcampos de estudio en política educativa con los posicionamientos epistemológicos o perspectivas epistemológicas?

Finalmente, el componente que hemos denominado Enfoque Epistemetodológico. Con el cual nos referimos al momento metodológico donde el investigador opta por una u otra metodología. No consideramos a los 'enfoques metodológicos' como meros instrumentos sea de recopilación, sea de análisis de la información. Sino como el "método del logos", esto es el modo de pensar el logos. Por tal razón preferimos hablar de epistemetodología, categoría en la que confluyen la presentación de método y la posición epistemológica del investigador. Consideramos que un enfoque metodológico posee una epistemología, pero a los efectos de la distinción de uso común, preferimos este último término: epistemetodología. No lo hacemos por una cuestión de mera sofisticación en los conceptos, sino más bien de precisión (Cfr. TELLO, 2011b).

\footnotetext{
${ }^{6}$ Una reflexión que surge al pensar las posibles combinaciones entre perspectiva epistemológica y posicionamiento da cuenta que estas son varias, podríamos mencionar a modo de ejemplo:

a) Perspectiva epistemológica: neo-marxismo, posición epistemológica: crítico radical. Para la misma perspectiva epistemológica del neo-marxismo el investigador puede tomar una posición epistemológica de la complejidad.

b) Perspectiva epistemológica: pos-estructuralista con una posición epistemológica: crítico radical. Para la misma perspectiva epistemológica del pos-estructuralismo se puede asumir una posición epistemológica posmoderna.

c) Perspectiva epistemológica: pluralismo con una posición epistemológica del neo-institucionalismo. Para la misma perspectiva epistemológica del pluralismo se puede asumir una posición epistemológica: hiperglobalista o escéptica, o crítico-radical.

Claro está que las combinaciones son válidas en la medida que el investigador sustente la relación entre perspectiva y posicionamiento. (Esta reflexión surgió a modo de ejercicio de una lectura crítica con el colega Jefferson Mainardes).

${ }^{7}$ Esto no significa que en este tipo de estudio no se hayan desarrollado o se estén desarrollando metodologías de análisis propias, pero no implican las presencia de un posicionamiento epistemológico.

${ }^{8}$ Entendiendo el método del logos en la clásica sentencia de Heráclito: 'uno es todo'. En este sentido la metodología es un componente lógico que no puede ser pensada de un modo aislado porque conforma la epistemología de la investigación.
} 
Es decir, debemos tener en cuenta que la metodología se convierte en una epistemetodología en la medida que está enhebrada a los otros componentes: Perspectiva epistemológica y Posición Epistemológica. Por sí sola y, por ejemplo a los efectos de la enseñanza son sólo metodologías, con mayor precisión conceptual nos podemos referir a la metodología como técnicas metodológicas. Técnicas que muchas veces se enseñan erróneamente como epistemologías, y en otros casos, como dijimos, sin sustento epistemológico. Bourdieu (2000, p. 62) es agudamente irónico respecto de esta predominancia explicando que se la toma como "serie de recetas o de preceptos que hay que respetar no para conocer el objeto sino para ser reconocido como conocedor del objeto". Teniendo en cuenta esta advertencia es necesario que la metodología en términos de investigación se convierta en epistemetodología, en tanto constituya un componente consistente de la red delo tres componentes que conforma el EEPE.

De este modo una investigación en política educativa puede ser analizada tratando de identificar estos tres componentes, los cuáles nos permiten también comprender la complejidad de una investigación propia del campo, esto es, no encontraremos sólo uno de los componentes en el tejido de la investigación sino que existirá una Perspectiva epistemológica, un Posicionamiento Epistemológico y un Enfoque Epistemetodológico, con mayor o menor coherencia y consistencia entre sí.

En síntesis, la cuestión que presentamos no es si la investigación y la proyección social que realizan las ciencias humanas y sociales contienen o no valores e ideología, sino la necesidad de indagar qué valores se encuentran en cada proceso y situación, cuál es su papel y cómo se podrán, y deberán - desde las diferentes visiones de realidad -, hacer explícitas en el proceso de investigación. Un principio para tener en cuenta es que no se puede eliminar la subjetividad y los valores de la ciencia y de sus usos técnicos; habría, en cambio, que esforzarse en hacerlos explícitos, para observar su aportación al resultado final de la actividad de investigación en política educativa. Con esta orientación, el investigador asume mayor libertad de elección de un curso de acción o de un método determinado pero, paralelamente, mayor es la responsabilidad (personal y profesional) por las consecuencias de su proceder y por el valor social de los resultados de sus investigaciones, en tanto posición política: “¿Hasta qué punto ese conocimiento contribuye o no a la posibilidad de construir una sociedad más democrática y más equitativa?” (LANDER, 2000, p. 54). 


\section{Posición epistemológica y vigilancia epistemológica en el campo de la política educativa}

\section{La política educativa como campo de estudio y su relación con las epistemologías de la política educativa}

Podríamos pensar en la posibilidad de precisar algunos rasgos de las epistemologías de la política educativa pero nunca considerar que se podría definir la "teoría de la política educativa" entendida esta última como una reducción epistemológica, dado que los planteos epistemológicos actuales no requieren de la "demarcación" disciplinar, lo cual responde más bien a estudios y concepciones clásicas desde la epistemología que distinguía por ejemplo ciencia-pseudocienciadisciplinas con un objeto de estudio delimitado y metodología propia, entrando en el falso camino del cientificismo; por el contrario, asumimos en el sentido de Wallerstein (2004), una postura a favor de la ciencia y el conocimiento y en contra del cientificismo.

De este modo, consideramos el campo de la política educativa como un espacio reticular, en tanto se define un campo de conocimiento desde la epistemología contemporánea, como la posibilidad de nuevas construcciones conceptuales que permiten la interacción múltiple y recíproca entre distintos campos de conocimiento. En términos de Gianella (2006, p. 79) "El retículo tiene una estructura doblemente compleja, ya que en la red se admiten integrantes que a su vez son redes". Así se produce la vinculación con categorías de la ciencia política, la sociología de la educación, la pedagogía y, donde la clave está en los múltiples objetos de estudio que construye la política educativa.

Pues, de algún modo las regulaciones internas del campo de la política educativa se estructuran en base a su relación con otros campos de estudio, y allí es donde nos encontraremos con las epistemologías de la política educativa ${ }^{9}$ en su estado puro - utilizando un término que pareciera contradictorio -, argumentamos "que pareciera contradictorio" porque su "estado puro" como campo tiene que ver con la complejidad de campos con los que se vincula.

Al ser un campo eminentemente de las ciencias sociales, se despliega desde la propia multiplicidad teórica, lo cual no cuestiona -desde nuestra perspectiva- su fortaleza epistemológica sino muy por el contrario es un espacio fértil y sólido. Dicha fortaleza y solidez se genera a partir de su diversidad para el análisis de las políticas educativas y producción del conocimiento científico. Entendiendo la rigurosidad epistemológica del campo como la capacidad de sostener la coherencia epistemológica en el proceso de investigación y no como hemos mencionado anteriormente con un objeto de estudio petrificado

\footnotetext{
${ }^{9}$ Aquí nos referimos específicamente al segundo uso del EEPE.
} 
y estático. Inferimos pues que, la presencia dinámica de los objetos de estudios de la política educativa como campo teórico no atentan contra la producción del conocimiento que se debe sostener en términos de solidez epistemológica.

Por tanto las epistemologías de la política educativa en Latinoamérica, puede venir en auxilio del status epistemológico del campo, tal como afirma Pedro Flores-Crespo (2012, p. 1) refiriéndose a los estudios epistemológicos en política educativa: "reflexionar sobre la forma en cómo se genera el conocimiento en este campo de estudio abona a su consolidación”.

\section{El posicionamiento y la vigilancia epistemológica}

Bourdieu et al. (2008) en 'el oficio del sociólogo' retoman la idea de los componentes inconscientes del investigador, que para los autores se encuentran naturalizados y esa naturalización no permite la reflexividad epistemológica sobre su propio proceso de investigación en tanto epistemologías de las política educativa: Perspectiva epistemológica, Posición Epistemológica y Enfoque Epistemetodológico. Considerando que todos los investigadores asumen una cierta visión del mundo y que de algún modo traen consigo.

Estas 'visiones' pueden ser nombradas de diversos modos, como "supuestos epistemológicos" (SIRVENT, 1999) o lo que en la perspectiva anglosajona se puede encontrar como ideas orientadoras que ayudarían a guiar la investigación: sensitiz̨ing concepts que permite, según Blumer (1954, p. 7) "una sensación general de referencia" o el desarrollo realizado por Bowen en Grounded Theory and Sensitizing Concepts (2006) o "presupuesto teórico" para Wainerman (2001). En fin, distintas categorías para nominar este proceso subjetivos del investigador.

De ningún modo rechazamos los supuestos con los que un investigador comienza a desarrollar su investigación "de hecho selecciona un tema y no otro, se hace algunas preguntas y no otras" (TELLO, 2011b, p. 229). McLuhan afirma en una célebre frase "No sé quién descubrió el agua por primera vez, pero estoy seguro de que no fueron los peces". En efecto, las personas insertas en un proceso de investigación naturalizan sus propios posicionamientos sin observar la necesidad de hacerlos explícitos y esto genera un dislocamiento entre realidad a ser investigada por el investigador y proceso de investigación, en términos de Bourdieu et al. (2008):

[...] el acto científico fundamental es la construcción del objeto: no vamos a la realidad sin hipótesis, sin instrumentos de construcción. Y cuando se le cree desprovisto de todo presupuesto, se le construye aún sin saber y, casi siempre, en este caso, de manera inadecuada. (BOURDIEU et al., 2008, p. 44). 
Los investigadores en políticas educativas muchas veces dan por supuesta su posicionamiento y perspectiva epistemológica. Siguiendo los postulados de Mainardes (2009) y Flores-Crespo (2008) se considera que con la presencia de autores y el uso de sus ideas se presenta una epistemología de la política educativa, y aquí es donde el EEPE advierte sobre la necesidad de la revisión de la coherencia y solidez de la investigación. Así, la vigilancia epistemológica en la investigación en el campo de la política educativa la entendemos como aquella práctica actitudinal por parte del investigador que debe estar siempre presente para sostener una reflexividad epistemológica en el proceso de investigación. La reflexividad se convierte en una exigencia propia del funcionamiento autónomo del campo de estudio de la política educativa. Por lo tanto la reflexividad epistemológica en términos de reflexión crítica del propio proceso de investigación a través del esquema analítico del EEPE debe contribuir, al menos, en dos aspectos: a) explicitación de supuestos previos ${ }^{10}$ (presupuestos teóricos, sensitizing concepts, supuestos epistemológicos, etc) b) explicitación del posicionamiento epistemológico, perspectiva epistemológica y enfoque epistemetodológico como un modo de "sistemas controles cruzados" para el propio investigador.

Consideramos que las dificultades de la reflexión crítica no se encuentran solo al momento de desarrollar la investigación, que en ocasiones hemos visto que contienen los componentes de una epistemología de la política educativa, sino en otra etapa compleja de la investigación: la difusión de los resultados de los resultados de investigación. Dado que allí, en la escritura de informes de investigación, publicación de papers o libros, muchas veces por una cuestión de espacio o porque el investigador está preocupado y ansioso por explicar sus resultados deja a un lado el desarrollo de su posicionamiento, perspectiva epistemológica y su enfoque epistemetodológico.

En fin, esto que le sucede al investigador debe ser nombrado, si no se explicita no habría reflexión sobre el proceso epistemológico, esto es: no habrá epistemología de la política educativa, asumiendo que obtendrá resultados de una investigación, sin conocer ni reflexionar "desde dónde" ni "para qué" investiga. Como hemos mencionado en la presentación otra de las dificultades consiste en no establecer la distinción entre la metodología y la epistemología, tomando a veces la primera como sinonimia de la segunda.

\section{Entre la técnica metodológica y las epistemologías de la política educativa}

Es necesario considerar en este hilvanado conceptual la necesidad de establecer algunas diferenciaciones en la estructura de una investigación en

\footnotetext{
${ }^{10}$ Para un desarrollo más específico sobre estas categorías véase Tello (2011b).
} 
política educativa. En este sentido, debemos considerar el planteo de Ball (2006) cuando se refiere a la investigación en política educativa señalando que en una investigación del campo se puede observar, por un lado, una "epistemología de superficie" como la relación que se establece entre conceptualizaciones, conducción, diseño y esquematización de una investigación. Es decir allí no encontramos una epistemología.

Por esta razón no puede dejar de tenerse en cuenta para la solidez y consistencia de la misma la "epistemología profunda" (BALL, 2006), esto es las formas de explicación y descripción del relato de la investigación. Lo que nosotros hemos denominado los componentes de la epistemología de la política educativa.

Las consideraciones vertidas por Ball (2006) son tomadas de un modo erróneo como pasos sucesivos, nos referimos a la falsa concepción del Paso I y del Paso II que se presentan en una investigación. Entendiendo el Paso I como el propio diseño: el recorte de un objeto de estudio, la selección empírica, la propuesta de objetivos de investigación. Lo consideramos erróneo porque como hemos señalado anteriormente allí existe un posicionamiento y una perspectiva epistemológica que consecuentemente se despliegan en el enfoque epistemetodológico. El Paso I no es una tarea meramente técnica o de "superficie" y, si así se lo tomase la dificultad se encontraría en cuanto a que muchas investigaciones se llevan a cabo desde la "epistemología de superficie", es decir muchas investigaciones en política educativa son investigaciones que sólo desarrollan prolijas cuestiones técnicas. Aquí se da un atravesamiento importante de la producción de conocimiento técnico-instrumental, reduciendo el proceso investigativo a una sucesión de etapas y fases que al cumplirlas se lograría rigurosidad científica, lo cual es completamente falso.

Es aquí donde debemos reflexionar acerca de que la metodología no es solo "una metodología", sino epistemetodología en términos de la epistemología de la política educativa. Esto es, la metodología - como hemos mencionado anteriormente - solo es un componente de una investigación. Ahora bien, cuando ese componente supera la epistemología de la superficie, esto es que posee un posicionamiento por parte del investigador en relación a los otros componentes de una investigación se observa una epistemología profunda: una epistemetodología. El posicionamiento del investigador sobre la metodología, en relación a los otros componentes de investigación es epistemetodología y si solo las presenta como "técnicas" es "epistemología de la superficie". La pregunta que cabe aquí es ¿desde qué epistemología se llevan a cabo los diseños y proyectos de investigación en políticas educativas? 
Teniendo en cuenta que como afirma Mainardes (2009):

Muchos investigadores no explicitan los supuestos teóricos que sustentan sus análisis $[\ldots]$ se observa el uso de ideas de un conjunto de autores (muchas veces de matrices epistemológicas distintas) para sostener el análisis. Eso torna difuso e inconsistente los fundamentos de las investigaciones. (MAINARDES, 2009, p. 7).

Aunque este no parecería ser solo un problema de nuestra región, FloresCrespo (2012) advierte que en Europa, - tomando las consideraciones de Thoenig -muchos estudios en el campo de la política carecen de rigor analítico y los trabajos son "en esencia, descriptivos y teóricamente inestables, semejan más estudios prácticos o informes de consultoría"; por lo tanto, "no producen mucho conocimiento nuevo ni información rigurosa o teorías sólidas" (THOENIG, 2004, p. 189).

En términos de Bourdieu (2000) es clave conocer desde "donde se habla", desde que lugar se posiciona el investigador. Invitar a pensar sobre las epistemologías de la política educativa consistiría en hacer explícitos los posicionamientos, perspectivas y enfoques de las investigaciones en política educativa. También debemos tomar la advertencia de Zemmelan (1998, p. 123) cuando explica que existe en la investigación social en la actualidad latinoamericana un "desequilibrio entre el extraordinario desarrollo técnico-metodológico y el escaso desenvolvimiento del pensamiento abstracto de las ciencias sociales en su expresión ya sea filosófica o bien epistemológica".

En fin, el EEPE surge como un auxiliar analítico para mantener la vigilancia epistemológica sobre los procesos de investigación en políticas educativas. Ahora bien, otro uso de EEPE es el que vienen desarrollando los investigadores de la Red Latinoamericana de Estudios Epistemológicos en Política Educativa ${ }^{11}$ (ReLePe) y que consiste en desarrollar estudios epistemológicos sobre política educativa, ya no como vigilancia epistemológica del propio proceso de investigación sino como investigación sobre investigaciones en políticas educativas (TELLO, 2011). Es decir, llevando a cabo una meta-análisis desde el plano de "otro" investigador en política educativa que analiza una investigación en política educativa llevada a cabo por otro investigador. Así el investigador toma una investigación, llevo a cabo el análisis con los componentes de la EEPE e intenta comprender la epistemología de esa investigación.

\section{Notas Finales}

Sin dudas que asistimos a un nuevo modo de comprender la realidad, cuyos efectos están cambiando radicalmente nuestra manera de conocer el mundo y, como si lo anterior no fuera suficiente, de relacionarnos con él. En este sentido

\footnotetext{
${ }^{11}$ Cfr. $<$ http://www.relepe.org $>$.
} 
debemos considerar que el campo de la política educativa cuyo continente son las ciencias sociales requiere de un nuevo espacio de reflexión epistemológico luego de la "tormenta neoliberal" (TORRES, 2008). Como dijimos, en Latinoamérica, comenzar a caracterizar las epistemologías de la política educativa puede venir en auxilio del status epistemológico del campo. Entendiendo, en términos de Gatman (2011) que:

Es una creencia ampliamente aceptada que las ciencias sociales tienen características idiosincrásicas distintivas de acuerdo a su origen nacional o regional. No suele hablarse de una química alemana como diferente a una química italiana o estadounidense, pero sí es posible pensar en una sociología francesa, una sociología anglo-americana o una sociología latinoamericana (GATMAN, 2011, p. 408).

Ahora bien, considerando este postulado la política educativa y los estudios epistemológicos en este campo, particularmente en nuestra región, tienden a desconocer los desarrollos conceptuales potentes que se producen en Gran Bretaña, EE. UU, Australia, entre otros. De este modo consideramos que el debate epistemológico en este campo teórico en Latinoamérica aún es escaso (Cfr. BALL; MAINARDES, 2011). Este es uno de los principales aportes que consideramos que puede realizar este trabajo: contribuir al fortalecimiento epistemológico del campo teórico, sin tener como horizonte la distinción entre ciencia, disciplina o subdisciplina. Por el contrario el fortalecimiento epistemológico del campo teórico se da en la medida que asume su multidisciplinariedad y complejidad interdisciplinaria en el desarrollo para la investigación en política educativa, que se manifiesta en su diversidad de posicionamientos y perspectivas (TELLO, 2009). También es necesario considerar que no se deben "copiar" epistemologías de otras latitudes, sino que Latinoamérica debe asumir el desafío de construir sus propias epistemologías, sin desconocer la potencia teórica de desarrollos en otras partes del mundo.

El EEPE intenta de algún modo proponer una mirada que contemple la realidad social que nos convoca a los investigadores desde la profundidad de la epistemología; una epistemología que debe ser parte de la realidad y contribuir a ella.

Desde nuestra perspectiva, las epistemologías de la política educativa contribuirán a que la claridad teórica y la reflexividad en torno a las propias producciones de política educativa generarían mayor precisión en los diálogos entre investigadores y en este sentido un fortalecimiento del campo. Teniendo en cuenta que existirán diversas epistemologías: la de la opresión, las hegemónicas o simplemente las de adhesión a sistemas políticos. Esto es en el sentido de Gewirtz (2007, p. 7) llevar a cabo una "reflexividad ética sobre el trabajo de investigación" o en términos de Bourdieu et al. (2008, p. 46) "la reflexividad exige 
la explicitación de los postulados de partida de toda práctica con pretensiones científicas, es un efecto de control epistemológico", en fin reflexionar sobre las posibles consecuencias epistemológicas es hacerlo en término de ética de la investigación, esto es: de qué forma ciertos trabajos legitiman determinadas epistemologías y no otras. Dado que consideramos que una epistemología es siempre una antropo-ética en el sentido de Morín (2001), en sus tres dimensiones: una ética de la solidaridad, de la comprensión y la ética del género humano. Allí entra en juego, de un modo clave, la vigilancia epistemológica, como un modo de comprender los procesos de investigación en una perspectiva ético-política. En tanto la epistemología es epistemología ética.

Si logramos avanzar en este camino, probablemente también, podamos pensar en una epistemología de la política educativa latinoamericana (Cfr. TELLO, 2010). En la cual la investigación en políticas educativas contribuya a fortalecer y desarrollar la justicia educativa y la consolidación democrática en nuestra América.

\section{Referencias}

BALL S. Policy sociology and critical social research: a personal review of recent education policy and policy research. British Journal of Educational Research, v. 23, n. 3, p. 257-274, 1997. DOI: $10.1080 / 0141192970230302$

Sociología das políticas educacionais e pesquisa crítico-social: uma revisão pessoal das políticas educacionais e da pesquisa em política educacional. Currículo sem Fronteiras, v. 6, n. 2, p. 10-32, jul./dez. 2006.

BALL, S. J.; MAINARDES, J. Introdução. In: BALL, S. J.; MAINARDES, J. (Orgs.). Políticas Educacionais: questões e dilemas. São Paulo: Cortez, 2011. p. 11-18.

BARRIGA, O.; HENRÍQUEZ, G. La presentación del objeto de estudio. Revista Cinta de Moebio, n. 17, p. 1-20, 2003. Disponible en: <http://www.facso.uchile.cl/publicaciones/ moebio/17/barriga.htm>. Acceso: 24 jul. 2009.

BLUMER, H. What is wrong with social theory? American Sociological Review, v. 19, n. 1, p. 3-10, 1954.

BOURDIEU, P. Los usos sociales de la ciencia. Buenos Aires: Nueva Visión, 2000. . et al. El oficio de sociólogo. Buenos Aires: Siglo XXI, [1973], 2008.

BOWEN, G. A. Grounded theory and sensitizing concepts. International Journal of Qualitative Methods, v. 5, n. 3, p. 1-9, 2006.

COX, C. Construcción política de reformas curriculares: el caso de chile en los noventa. Profesorado. Revista de currículum y formación del profesorado, España, v. 10, n. 1, p. 1-24, 2006.

FLORES-CRESPO, P. Análisis de política pública en educación: línea de investigación. México: UIA-INIDE, 2008. (Cuaderno Investigación, n. 5). 
Un acercamiento a la epistemología de las políticas educativas: conceptos, unidad de análisis y teoría. 2012. Mimeo.

GATMAN, E. La productividad científica argentina en ciencias sociales: economía, psicología, sociología y ciencia política en el CONICET (2004-2008). Revista Española de Documentación Científica, Madrid, v. 34, n. 3, p. 408-425, jul./sep. 2011.

GEWIRTZ, S. A reflexividade ética na análise de políticas: conceituação e importância. Práxis Educativa, Ponta Grossa, v. 2 , n. 1, p. 7-12, jan./jun. 2007.

GIANELLA, A. Las disciplinas científicas y sus relaciones. Anales de la educación común, Buenos Aires, v. 2, n. 3, p. 74-83, abr. 2006.

GLASSER, B.; STRAUSS, A. The discovery of grounded theory: strategies for qualitative research. Nueva York: Aldine, 1967.

HABERMAS, J. Conocimiento e interés. Madrid: Taurus, 1981.

Ciencia y técnica como ideología. Madrid: Tecnos, 1984.

LANDER, E. “¿Conocimiento para qué? ¿Conocimiento para quién?”, In: CASTROGÓMEZ, S. (Ed.). La reestructuración de las ciencias sociales en América Latina. Bogotá: Instituto PENSAR, 2000. p. 49-70.

MAINARDES, J. Análise de políticas educacionais: breves considerações teóricometodológicas. Contrapontos, Itajaí, v. 9, n. 1, p. 4-16, jan./abr. 2009.

MAINARDES, J.; SANTOS, M.; TELLO, C. Análise de políticas: fundamentos e principais debates teórico-metodológicos. In: BALL, S. J.; MAINARDES, J. (Orgs.). Políticas Educacionais: questões e dilemas. São Paulo: Cortez, 2011. p. 143-172.

MORIN, E. Los siete saberes necesarios para una educación del futuro. México: UNESCO, 2001.

POPKEWITZ, T. S. Sociología política de las reformas escolares: el poder-saber en la enseñanza, la formación del profesorado y la investigación. Madrid: Morata, 1994.

SIRE, J. The universe next door: a basic worldview catalogue. Downers Grove, IL: InterVarsity Press, 2004.

SIRVENT, M. T. Problemática actual de la investigación educativa. Revista del Instituto de Investigaciones en Ciencias de la Educación, Buenos Aires, v. 8, n. 14, p. 64-75, 1999.

SOUSA SANTOS, B. de. La Sociología de las ausencias y la sociología de las emergencias: para una ecología de saberes. In: Renovar la teoría crítica y reinventar la emancipación social (encuentros en Buenos Aires). Buenos Aires: CLACSO, 2006. p. 13-41.

La reinvención del conocimiento y la emancipación social. Buenos Aires: Siglo XXI, CLACSO, 2009.

TELLO, C. Las epistemologías de la política educativa. In: CONGRESO INTERNACIONAL EDUCACIÓN, LENGUAJE Y SOCIEDAD: "LA EDUCACIÓN EN LOS NUEVOS ESCENARIOS SOCIOCULTURALES”, 2., 2009, La Pampa. Actas... La Pampa, Argentina, 2009. 
Política educativa y fraternidad en América Latina. Notas epistemológicas. In: BARRENECHE, O. (Coord.). Estudios sobre fraternidad. Buenos Aires: Editorial Ciudad Nueva, 2010. p. 187-201. (Serie Política y Sociedad).

Las epistemologías de la política educativa: notas históricas y epistemológicas sobre el campo, 2011a. Mimeo.

. El objeto de estudio en ciencias sociales: entre la pregunta y la hipótesis debates sobre las decisiones epistemológicas en un proyecto de investigación. Revista Cinta de Moebio, n. 42, p. 225-242, 2011b. Disponible en: < http:/ /www.revistas.uchile.cl/index.php/ CDM/article/viewArticle/18162/19020>. Acceso: 12 fev. 2012.

TELLO, C.; MAINARDES, J. La posición epistemológica de los investigadores en Política Educativa: debates teóricos en torno a las perspectivas neo-marxista, pluralista y posestructuralista. Archivos Analíticos de Políticas Educativas, v. 20, n. 9, p. 1-37, 2012. Disponible en: <http://epaa.asu.edu/ojs/article/view/988>. Acceso: 24 mar. 2012.

THÖENING, J. C. La actualización del conocimiento en el campo de la administración y las políticas públicas. In: PARDO, M. del C. (Comp.). De la administración pública a la gobernanza. México: COLMEX, 2004. p. 183-216.

TORRES, C. A. Critical theory and political sociology of education: arguments. In: POPEKWITZ, T.; FENDLER, L. (Eds.). Critical theory in educational discourse. New York: Routledge, 1999. p. 87-116.

Después de la tormenta neoliberal: la política educativa latinoamericana entre la crítica y la utopía. Revista Iberoamericana de Educación (OEI), v. 48, p. 207-229, 2008. VITAR, A. Políticas de educación: razones de una pasión. Buenos Aires: Miño y Dávila, 2006.

WAINERMAN, C. "Acerca de la formación de investigadores en ciencias sociales” Errores comunes en la formulación de investigaciones sociales. In: WAINERMAN, C.; SAUTU, R.

La trastienda de la investigación. Argentina: Lumiere, 2001. p. 25-34.

WALLERSTEIN, I. Las incertidumbres del saber. Barcelona: Gedisa, 2004.

ZEMELMAN H. Crítica, epistemología y educación. Revista de Tecnología Educativa, Santiago de Chile, v. 2, n. 13, p. 119-131, 1998.

Recebido em 28/01/2012

Versão final recebida em 24/04/2012

Aceito em 26/04/2012 